\title{
Study of the interface friction between mesh and rock surface in drapery systems for rock fall hazard control
}

\author{
Ivan Gratchev ${ }^{1 *}$, Dong Hyun Kim², and Matthew Chung ${ }^{3}$ \\ 1) Senior Lecturer, Griffith School of Engineering, Griffith University, Australia. \\ *Corresponding author. Email: ivangratchev@gmail.com \\ 2) PhD candidate, Griffith School of Engineering, Griffith University, Australia \\ 3) Graduate site engineer at Lend Lease, QLD, Australia
}

\begin{abstract}
Although drapery systems are commonly used to control rock fall hazard on steep slopes, some elements of their design such as the interface friction between the mesh and ground surface are still difficult to quantify in practice. This technical note presents a new test procedure designed to study the mechanism of rock-mesh interaction in the laboratory. A series of tilt tests and tests with increasing loads were performed to study the effects that number and type of rock-mesh contacts, slope angle, and mesh characteristics had on mesh-rock interaction. The obtained data indicated that the process of interlocking between the mesh and rocks could increase the mesh's resistance to failure as well as decrease the force acting on the anchors during accumulation of rock debris or snow. Some changes to the current guidelines regarding the selection of interface friction angle are also suggested.
\end{abstract}

Key words: rock fall; drapery system; interface friction; laboratory test 


\section{Introduction}

Rock falls are natural phenomena that cause significant damage to structures and transportation routes. To cope with this hazard, several rock fall protection methods such as benches (Alejano et al. 2007), catch ditches (Pierson et al. 2001) and flexible catch fences (Peila et al. 1998) have been developed and employed on steep slopes. In recent years, draped mesh systems have also become a popular method to control rockfall on actively eroded slopes (Bertolo et al. 2009; Giacomini et al. 2012). It generally consists of a steel mesh draped over a steep slope, which is suspended from upslope anchors (Shu et al. 2005). The design of these systems is dictated by engineering judgement and experience, and it primarily depends on the slope conditions and available funding. These days, engineers may consider different support options, including top anchors only or a series of anchors, which are fixed in the field of the mesh to also improve the slope stability (Bertolo et al. 2009).

The use of top anchors only as shown in Fig. 1 has some advantages as it results in lower installation cost and simplified maintenance which involves the removal of rock debris from the base of the slope. Muhunthan et al. (2005) and Shu et al. (2005), who reviewed the performance of drapery systems at several sites in North America, noted that these drapery systems generally functioned well. However, the investigators also reported a few global failures of the whole structure due to extra loads from falling rocks or snow accumulation. These failures highlighted the uncertainties in the design procedure that still need to be addressed. For example, the interface friction between the mesh and the rock surface is a parameter that contributes to the system support by reducing the force that is transferred to the anchors during extra loads. However, it is also a parameter that is rather difficult to quantify in practice as it varies greatly across the slope due to changes in surface conditions. The variety of mesh-rock contacts such as the surface (or interface) friction (Fig. 2a) and the degree of interlocking (Fig. 2b) also add to the complexity of the task. Some guidelines 
regarding the selection of the interface friction based on the type of slope surface were suggested by Sasiharan et al. (2006). For example, for slopes with very irregular and undulating surfaces, the interface friction angle can be assumed to be about $60^{\circ}$, while for slopes with planar and smooth surfaces, the friction can be in the range of $25-35^{\circ}$. However, these guidelines seem rather general, which may lead to overdesign and extra cost. It is clear that more research needs to be conducted to improve our knowledge about the mechanisms of mesh-surface interaction.

This work seeks to investigate the mechanism of mesh-surface interaction and clarify the effect of interface friction and interlocking on this process. To achieve this goal, a "rock wall” was built to mimic the mesh-surface interaction in the laboratory. A series of a) tilt tests and b) tests with increasing loads acting on the mesh were performed to study the effect of rock shape, slope inclination, and different mesh types on the friction between the rock surface and mesh. This technical note presents and discusses the obtained results.

\section{Interface friction between the mesh and rocks}

\subsection{Experimental setup}

The experimental setup consisted of the "rock wall" (top part) and the base (Fig. 3). The top part included a 1.2 (width) x 2.0 (length) m foamply plate with a thickness of $17 \mathrm{~mm}$ and a set of concrete blocks of different shapes (Fig. 4). The base part was made of wooden braces and sturdy metal legs to provide support to the whole structure. As the plate was connected to the base through the system of hinges, it was possible to incline the plate at different angles by means of a "block and tackle”. 
The shape of rocks determines the surface roughness including the number and type of mesh-rock contact points. To create different "rock surfaces”, several octahedron, round, and square blocks were made of concrete and attached to the plate in different patterns. The shape and size of these blocks are detailed in Fig. 4. The strength of concrete was obtained from three unconfined compression tests on cylindrical samples (diameter of $100 \mathrm{~mm}$, length of $200 \mathrm{~mm}$ ), resulting in an average UCS value of $50 \mathrm{MPa}$. The octahedron-shaped blocks were used to produce the interlocking type of contact between the mesh and blocks (Fig. 5a) while the round-shaped blocks only enabled the surface friction as shown in Fig. 5b. The square blocks could produce both types of mesh-rock interaction (Fig. 5c) and were considered as “intermediate" between the octahedron and round shapes.

Maccaferri double-twisted hexagonal mesh without (it is referred to as "DT" in this work) and with PVC coating (“DTC”), and Geobrugg chain link (single-twisted) mesh (“ST”) were used to investigate the effect of mesh fabric on the mesh-rock interaction. The details of each mesh are given in Fig. 6. The following features are noted: a) both DT and DTC were more rigid than ST, b) the opening size of DT $(\phi=78 \mathrm{~mm})$ and DTC ( $\phi=76 \mathrm{~mm})$ was greater than the one of ST ( $\phi=67 \mathrm{~mm})$, and c) due to the PVC coating, the wire diameter of DTC was slightly greater (3.7 mm) compared to ST (3 mm) and DT (2.7 mm).

\subsection{Test procedure}

To study the interface friction between the mesh and rock surface, a series of tilt tests were performed. The top plate was first lowered to its initial, horizontal position and blocks of the same shape were attached to the plate. For each next testing setup, the number of blocks increased (by twofold) to produce a greater number of mesh-block contact points. From the authors' experience, the largest number of contact points in each arrangement was made 
when the blocks were placed further apart from each other. Considering the above, the following block arrangements were used (Fig. 7): 2 blocks - (location B2 and B15), 4 blocks - (B1, B4, B13, and B16), and 8 blocks - (B1, B4, B5, B8, B9, B12, B13, and B16).

The mesh was placed on the top of the blocks, and the plate was then slowly lifted by the block and tackle system. The tilt angle ( $\alpha$ ) was monitored during testing by means of an electrical inclinometer, and the critical tilt angle at which the mesh failed (e.g., the mesh began to slide) was recorded. Different block shapes (octahedron, round, and square) and types of mesh (DTC, DT, and ST) were used. For each setup, the test was repeated 5 times and the average value was reported.

\subsection{Results and discussion}

Results from a series of tilt tests are summarized in Fig. 8 for DTC (a), DT (b), and ST (c). Regardless of the type of mesh, the following relationships were observed: a) an increase in the number of blocks (e.g., increase in the number of mesh-block contact points) correlated with greater critical tilt angles; b) the octahedron-shaped blocks produced higher tilt angles while the round-shaped blocks were associated with lower values. The large discrepancy between the octahedron- and round-shaped blocks can be attributed to the different types of contacts which were developed between the blocks and mesh. Visual observations during testing revealed that for the round-shaped blocks, the mesh was only "touching" the surface of each block (Fig. 5b), producing "surface" or interface friction between the mesh and the block. However, in the case of the octahedron-shaped blocks, the mesh was also "sitting" on the protruding parts of the blocks as shown in Fig. 5a. This “interlocking” between the mesh and blocks appeared to be the major factor leading to significantly higher values of the tilt angel at failure. It is noted that some degree of interlocking was also observed for the square- 
shaped blocks; however this type of contact was much less pronounced as compared to the octahedron-shaped blocks.

The data from Fig. 8 also indicate that a) DTC and DT mesh produced larger tilt angles then ST mesh, and b) DTC yielded the highest values of tilt angle. The first finding can be attributed to the larger mesh opening size of DTC and DT compared to ST (Fig. 6), which provided greater contact area between the mesh and blocks, and thus greater friction. The highest values of tilt angle obtained for DTC mesh can be due to the presence of PVC coating. The available literature (Rabbat and Russel, 1985; Molina Pombo, 2008) suggests that the coefficient of static friction between concrete and PVC material is generally higher (0.69) than the one between concrete and steel (0.57).

Considering the mesh as a free body on an inclined surface, it will tend to slide due to the gravity force (F) as the tilt angle increases. The friction mobilized between the mesh and the rock surface will produce a resistance force $\left(\mathrm{F}_{\text {res }}\right)$ preventing the mesh from sliding. The friction angle, which determines the magnitude of $F_{\text {res}}$, will reach its maximum value before the mesh begins sliding. Applying this concept to the tests with round-shaped blocks (where no interlocking was observed), the interface friction angle equal to the critical tilt angle $\left(\phi=\alpha_{r}\right)$ can be obtained. For the octahedron-shaped blocks, in addition to the interface friction, the interlocking between the mesh and the blocks also contributed to the resistance force, resulting in greater values of tilt angles $\left(\alpha_{0}\right)$ at failure. Therefore it can be assumed that the resistance force consisted of two components such as friction $\left(\mathrm{F}_{\mathrm{fr}}\right)$ and interlocking $\left(\mathrm{F}_{\text {int }}\right)$ force.

Considering the interface friction $\left(\phi=\alpha_{\mathrm{r}}\right)$, which was obtained from the tests with roundshaped blocks, the value of the interlocking force can be calculated using Eq. 1.

$$
F_{\text {int }}=F_{\text {res }}-F_{f r}=m \cdot g \cdot \sin \alpha_{o}-m \cdot g \cdot \cos \alpha_{o} \cdot \tan \alpha_{r}
$$


where, $\mathrm{m}$ - mass of the mesh, $\alpha_{0}$ - critical tilt angle obtained for round-shaped blocks, and $\alpha_{r}-$ critical tilt angle obtained for the octahedron-shaped blocks.

For example, for DTC mesh, a critical tilt angle of $26.6^{\circ}$ and thus the interface friction angle of $26.6^{\circ}$ were obtained for 4 round-shaped blocks (Fig. 8a). A larger tilt angle of $53.5^{\circ}$, which was obtained for 4 octahedron-shaped blocks, suggested that in addition to the friction force, there was significant amount of interlocking developed between the mesh and the blocks. Using Eq. 1, the interlocking force was found to be $26.0 \mathrm{~N}$ (Fig. 9a).

To estimate the extent to which $F_{\text {int }}$ contributes to the maximum value of $F_{\text {res }}$, the ratio between these two parameters $\left(\mathrm{F}_{\text {int }} / \mathrm{F}_{\text {res }}\right)$ was obtained (in \%) and plotted in Fig. 9b. It is evident from this figure that the interlocking component plays an important role in the meshrock interaction, especially for DTC and DT mesh where the interlocking force exceeded the interface friction component, contributing more than 50\% to the overall mesh resistance against failure.

\subsection{Practical significance}

1) In an attempt to establish relationships between the lab data and real-life performance of mesh, several case studies from Muhunthan et al. (2005) and Shu et al. (2005) where the failure of mesh system occurred due to snow accumulation are summarized in Table 1 . The back-calculated values of the interface friction angle, which was mobilized at the time of failure, were found to be in a range of $32-40^{\circ}$. However, it wasn't clear from the case description whether the reported values also included the interlocking component. It is interesting to note that this range seems to be slightly higher than the values of tilt angle obtained for the round-shaped blocks (no interlocking) but correlates with the results obtained 
for the square-shaped blocks where some occasional interlocking between the mesh and blocks was observed.

2) Current guidelines (Muhunthan et al., 2005; Sasiharan et al., 2006) suggest selecting the interface friction of $60^{\circ}$ for slopes with very irregular and undulating surfaces; that is, when more mesh-rock contact points are expected. The results of tilt tests with octahedron-shaped blocks revealed that the maximum tilt angle at which the mesh failed indeed could be as high as $60^{\circ}$. However, further analysis of the test results indicated that the actual interface friction between the mesh and rock was much lower, in the range of $25-30^{\circ}$, depending on the number of blocks used. In fact, it was the interlocking that resulted in such high values of critical tilt angles. Technically speaking, the interface friction angle of $60^{\circ}$ is significant overestimation; and the range of $25-30^{\circ}$ is recommended. However, an engineer will also need to estimate the amount of interlocking that can occur between the mesh and the rock based on the slope conditions. The following part of this paper will seek to provide more details regarding the interlocking process.

\section{Interlocking between the mesh and rocks}

The results from tilt tests revealed that the interface friction can be accompanied by interlocking between the mesh and rocks. From a practical point of view, it is useful to investigate the extent to which the interlocking can reduce the load that is transferred to the anchoring system. To achieve this goal, a series of tests using the procedure described below were performed.

\subsection{Test procedure}


The "rock wall” was inclined at a certain angle $(\beta)$ and kept stable by means of the angle brace (Fig. 10). Four slope angles of $60^{\circ}, 70^{\circ}, 80^{\circ}$, and $90^{\circ}$ were tested. Blocks of the same shape were attached to the plate in the following arrangements (Fig. 7): 2 blocks - (B9 and B12), 4 blocks - (B9, B12, B13, and B16), and 8 blocks - (B9-B16). To simulate the anchormesh system, the mesh was attached to the load cell which was fixed at the top of the plate (Fig. 10). This setup made it possible to measure the force which was generated by the additional loads. A series of dead loads (L) were applied to the bottom end of the mesh in a step-like fashion: 5.5, 11.0, 22.0, and $33.0 \mathrm{~kg}$. The acquired experience showed that the load of $33 \mathrm{~kg}$ was close to the maximum value that the "rock wall" could safely support. The force (F) that resulted from the application of load (L) was computed using Eq. 2 and compared to the force $\left(F_{m}\right)$ measured by means of the load cell. As $F_{m}$ was smaller than $F$, the difference between these two values was referred to as the force reduction $\left(\mathrm{R}_{\mathrm{f}}\right)$ and defined as shown in Eq. 3. This parameter $\left(\mathrm{R}_{\mathrm{f}}\right)$ was attributed to the interface friction and interlocking that mobilized between the mesh and blocks after the application of load. For example, higher values of $R_{f}$ (e.g., the larger difference between $F$ and $F_{m}$ ) were associated with greater friction and interlocking between the mesh and the blocks.

$$
\begin{aligned}
& F=(m+L) \cdot g \cdot \sin \beta \\
& R_{f}(\%)=\frac{F-F_{m}}{F} \cdot 100
\end{aligned}
$$

where, $\mathrm{m}$ - mass of mesh, $\mathrm{L}$ - load applied to the mesh, and $\beta$ - the angle at which the foamply plate was inclined.

\subsection{Test results and discussion}


Results from a series of tests with 2 (a) and 8 (b) octahedron-shaped blocks at different slope angles are plotted in Fig. 11 to demonstrate the effect of mesh-rock contacts on the force reduction $\left(\mathrm{R}_{\mathrm{f}}\right)$. It is evident from this figure that regardless of the slope inclination 1 ) an increase in the applied force (F) correlates with increasing values of $R_{f}$, and 2) a greater number of mesh-rock contact points (e.g., number of blocks used) leads to higher values of force reduction. It is also clear from the plots that higher values of $R_{f}$ were obtained for the slope angles of $70^{\circ}$ and $80^{\circ}$. At a slope angle of $90^{\circ}$, the mesh was hanging parallel to the rock wall with very limited mesh-rock interaction, which resulted in very low values of $\mathrm{R}_{\mathrm{f}}$. It is interesting to note that for a slope angle of $60^{\circ}$, the effect of rock-mesh interaction on the resistance force also decreased. Visual observations revealed that interlocking was mobilized to a lower level compared to that observed for slope angles of $70^{\circ}$ and $80^{\circ}$.

As the largest difference between $\mathrm{F}$ and $\mathrm{F}_{\mathrm{m}}$ was observed for the slope angle of $70^{\circ}$, further analysis was conducted to better understand the reason for such high values of $R_{\mathrm{f}}$. Figure 12 compares the results obtained for the round-shaped (a) and octahedron-shaped (b) blocks at the slope angle of $70^{\circ}$. Depending on the number of blocks, the force reduction in the tests with round-shaped blocks varied only from $10 \%$ to $15 \%$ while for the octahedron-shaped blocks, the difference ranged up to $50 \%$.

The octahedron-shaped blocks produced larger values of $R_{f}$, which can be attributed to the interlocking between the mesh and the blocks. Similar to the tilt test data, no interlocking was observed between the mesh and round-shaped blocks. This indicates that for the roundshaped blocks, the reduction in the force during the application of load was only caused by the interface friction. This assumption was used to calculate the interface friction using Eq. 4.

$$
\phi=\tan ^{-1}\left(\frac{F-F_{m}}{(m+L) \cdot g \cdot \cos \beta}\right)
$$


The relationships between the interface friction angle $(\phi)$ and the applied force $(F)$ for the round-shaped blocks are shown in Fig. 13a. As can be seen in this figure, as F increases, the interface friction angle mobilized between the mesh and round-shaped blocks also increases. In addition, the greater number of mesh-rock contacts leads to greater values of the interface friction angle.

For the octahedron-shaped blocks, the interlocking force $\left(\mathrm{F}_{\text {int }}\right)$ was estimated using Eq. 5 while the obtained results were plotted in Fig. $13 \mathrm{~b}$ as the $\mathrm{F}_{\text {int }} / \mathrm{F}$ against $\mathrm{F}$.

$$
F_{\text {int }}=F-F_{m}-(m+L) \cdot g \cdot \cos \beta \cdot \tan \phi
$$

It is clear from this figure that as the load increases, the contribution of the interlocking component to the force reduction also increases. It is evident that a greater number of contacts results in increasing interlocking effect. The data in Fig. 13b also indicate that for the range of loads used in the study, the interlocking effect can reduce the force acting on the anchor by $10-30 \%$, depending on the number of mesh-block contact points.

\section{Conclusions}

In this work, a new test procedure was used to study the mechanism of mesh-rock interaction. Based on the obtained results, the following conclusions can be drawn:

- The interface friction is typically accompanied by an interlocking effect which occurs between the mesh and rock surface, especially when the rocks have irregular shapes (such as octahedron or square in this study).

- The effect of mesh-rock interaction on the resistance force reaches its maximum at slope angles of 70 and $80^{\circ}$ and drops as the slope angle decreases to $60^{\circ}$. 
- Maccaferri double-twisted mesh with a large opening size and PVC coating can produce a larger resistance force against failure.

- The use of an interface friction angle of $60^{\circ}$ recommended by the current guidelines for irregular rock surfaces may be an overestimation. The range of $25-30^{\circ}$ for the interface friction angle combined with an estimation of the interlocking effect based on the slope surface is suggested.

\section{Acknowledgements}

The authors thank Mr. Jeroen Berends from Geo Inventions Consulting Services, Brisbane, for his invaluable help with this research. The authors also acknowledge the help from $\mathrm{Mr}$ Filbert Ndayishimiye with performing the tilt tests.

\section{References}

Alejano A, Pons B, Bastante F, Alonso E, Stockhausen H (2007) Slope geometry design as a means for controlling rockfalls in quarries. Int J Rock Mech Min Sci 44(6): 903-921

Bertolo P, Oggeri C, Peila D (2009) Full-scale testing of draped nets for rockfall protection. Can Geotech J 46 (3): 306-307

Giacomini A, Thoeni K, Lambert C, Booth S, Sloan SW (2012) Experimental study on rockfall drapery systems for open pit highwalls. International Journal of Rock Mechanics \& Mining Science 56: 171-181

Molina Pombo JC (2008) Mechanical Characterization of Fabrics for Inflatable Structures. Master Thesis, West Virginia University. UMI Dissertations Publishing 
Muhunthan B, Shu S, Sasiharan N, Hattamleh OA, Badger TC, Lowell SM et al (2005) Analysis and design of wire mesh/cable net slope protection, Washington State Transportation Center (TRAC) Report No. WA-RD 612.1

Peila D, Pelizza S, Sassudelli F (1998) Evaluation of behaviour of rock fall restraining nets by full scale tests. Rock Mech Rock Eng 31: 1-24

Pierson LA, Gullixson CF, Chassie RG (2001) Rockfall catchment area design guide. Final report SPR-(032) Metric Edition. Oregon Dept Transp \& FHWA http://www.oregon.gov/ODOT/TD/TP_RES/docs/Reports/RokfallCatchAreaDesMetric.pdf

Rabbat BJ, Russell HG (1985) Friction coefficient of steel on concrete or grout. Journal of Structural Engineering 111 (3): 505-515

Sasiharan N, Muhunthan B, Badger TC, Shu S, Carradine DM (2006) Numerical analysis of the performance of wire mesh and cable net rockfall protection systems. Eng Geol 88: 121132

Shu S, Muhunthan B, Badger TC (2005) Snow loads on wire mesh and cable net rockfall slope protection systems. Eng Geol 81: 15-31 
Figure 1. A view of drapery mesh and anchors system.

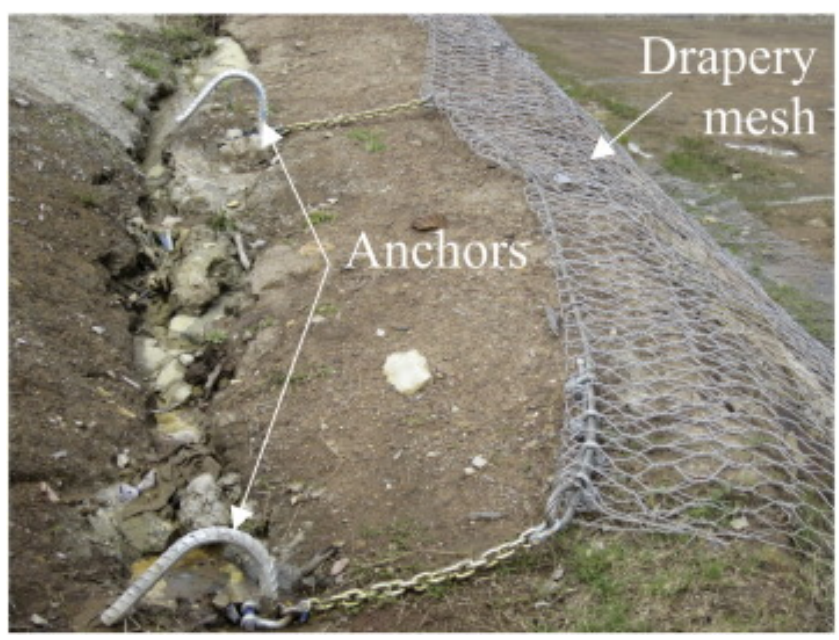

Figure 2. Types of mesh-rock contacts: a) interface friction, and b) interlocking between the mesh and rock

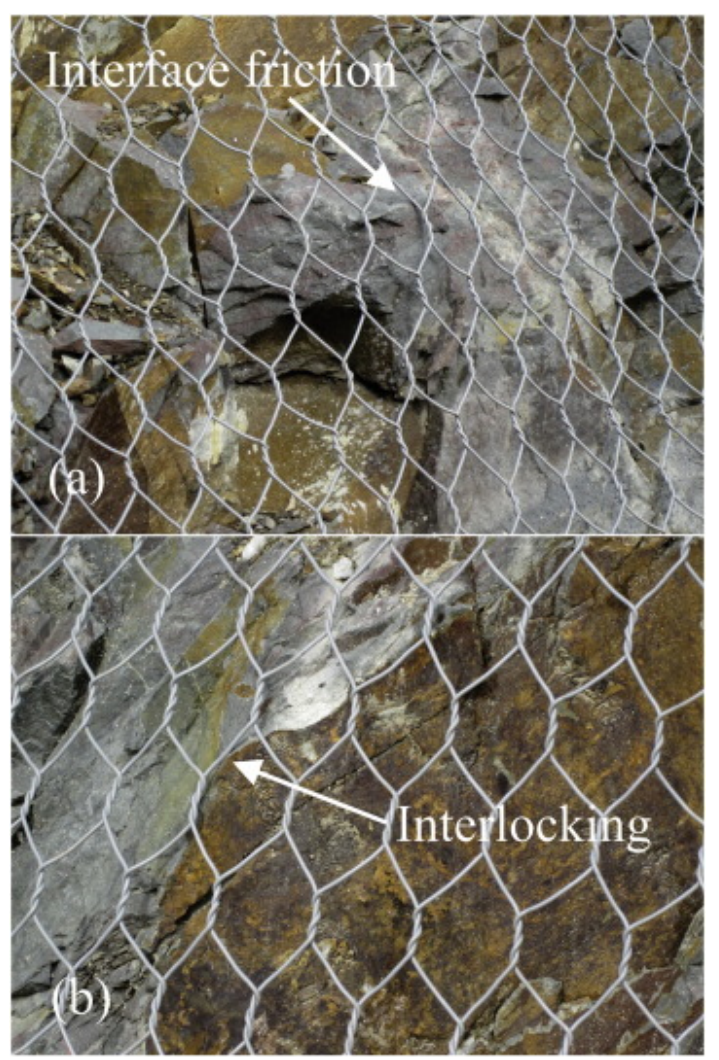


Figure 3. Experimental setup of tilt tests.

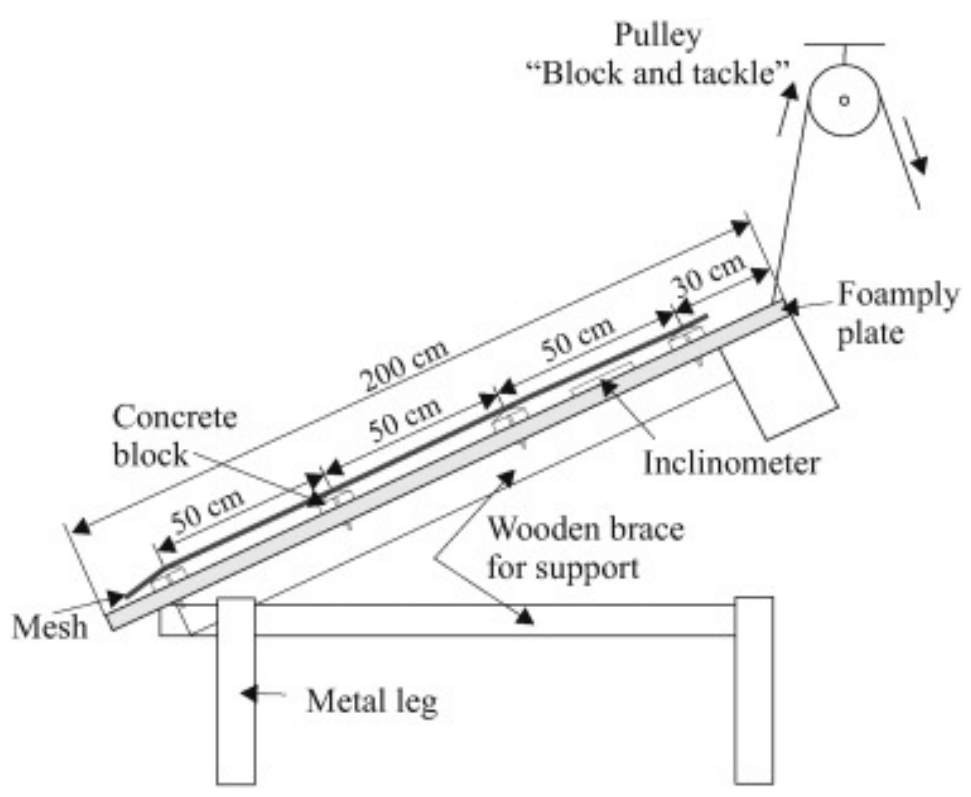

Figure 4. Concrete blocks of octahedron (a), round (b), and square (c) shapes.

a)

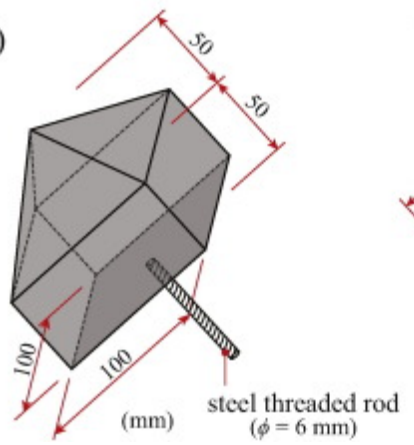

b)

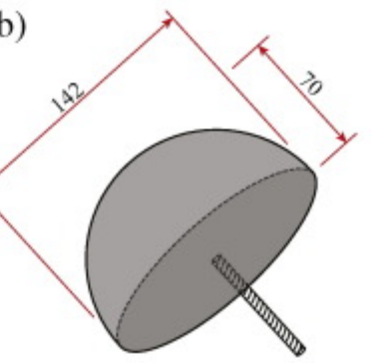

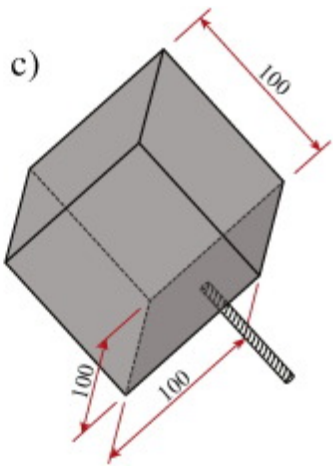


Figure 5. Mesh-block interaction observed for a) octahedron-shaped blocks (“interlocking), b) round-shaped blocks (surface friction), and c) square-shaped blocks.

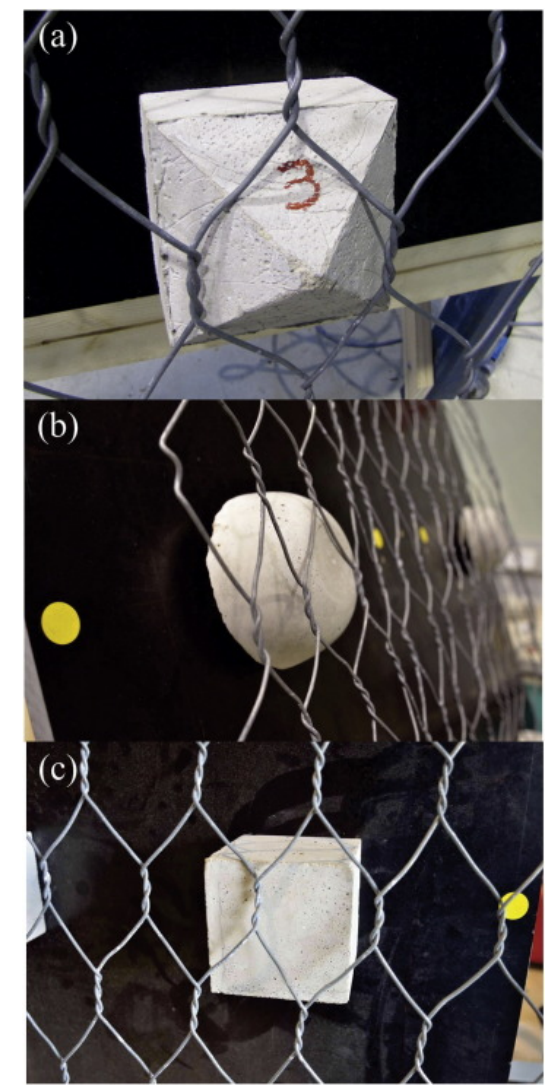

Figure 6. Types of mesh used: a) Maccaferri double-twisted mesh (DT), b) Maccaferri double-twisted mesh with PVC coating (DTC), and c) Geobrugg chain link (single-twisted) mesh (ST).

a)

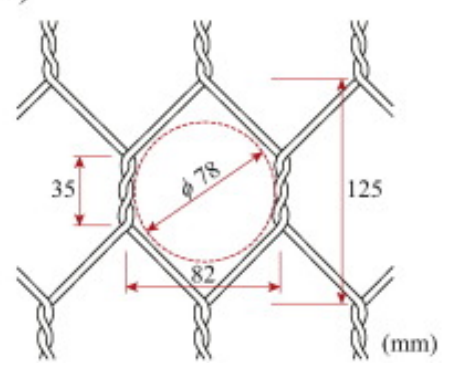

b)

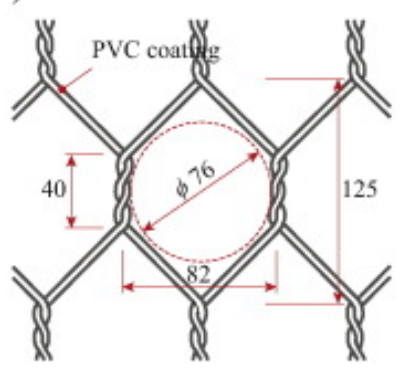

c)

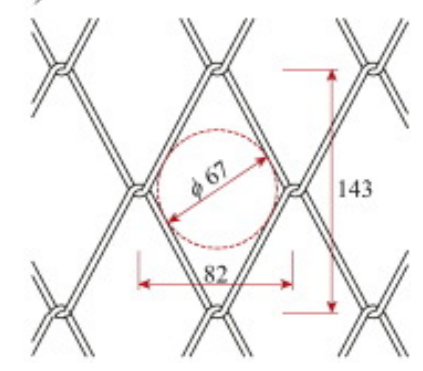


Figure 7. A front view of the "rock wall” with the location of concrete blocks.

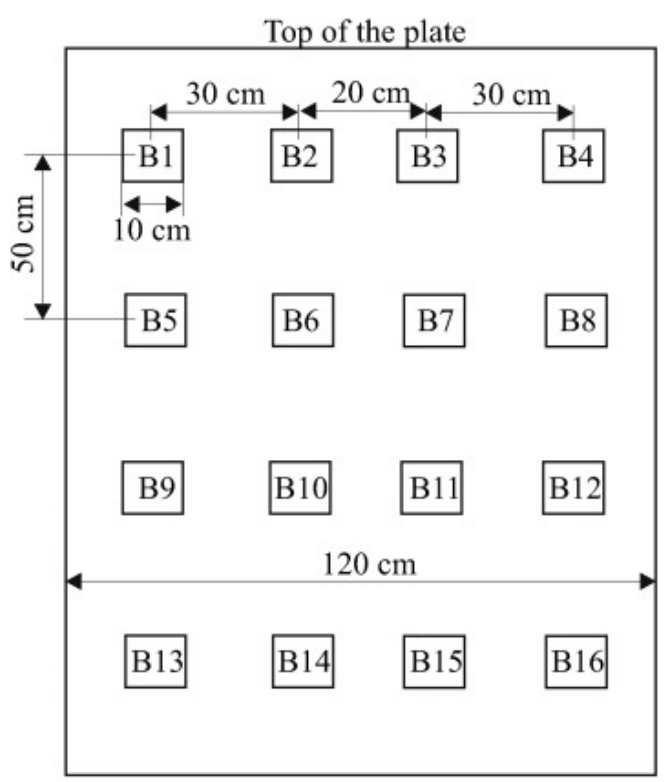

Figure 8. Results of tilt tests with different numbers and types of blocks obtained for (a) Maccaferri double-twisted mesh with coating (DTC); b) Maccaferri double-twisted mesh (DT), and c) Geobrugg chain link mesh (ST).
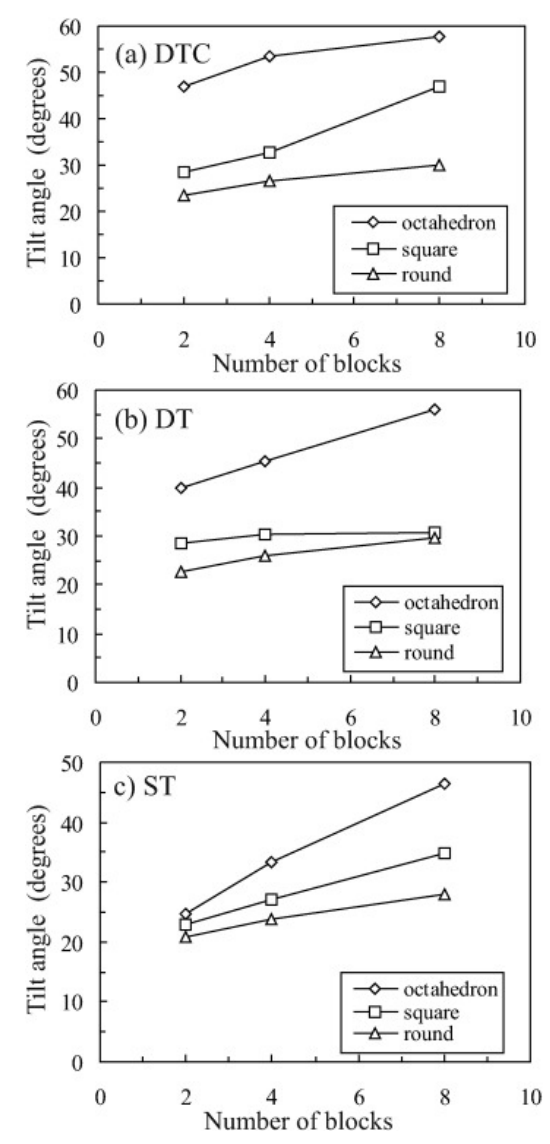
Figure 9. Results of tilt tests with octahedron-shaped blocks and different types of mesh. The relationship between the number of blocks and the interlocking force $\left(\mathrm{F}_{\text {int }}\right)(\mathrm{a})$, and the ratio between the interlocking $\left(\mathrm{F}_{\text {int }}\right)$ and resistance $\left(\mathrm{F}_{\text {res }}\right)$ forces $(\mathrm{b})$.
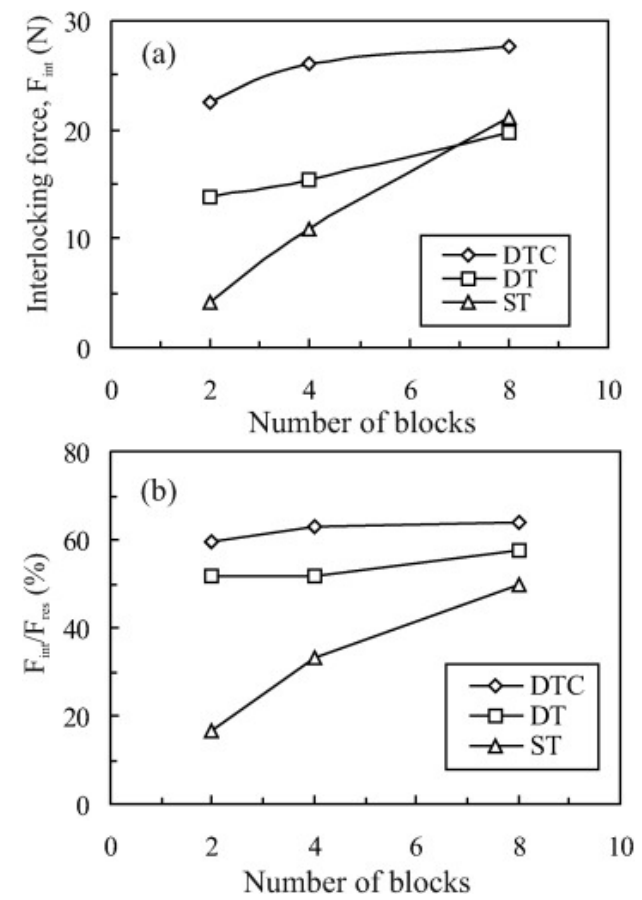

Figure 10. Experimental setup of tests with increasing loads.

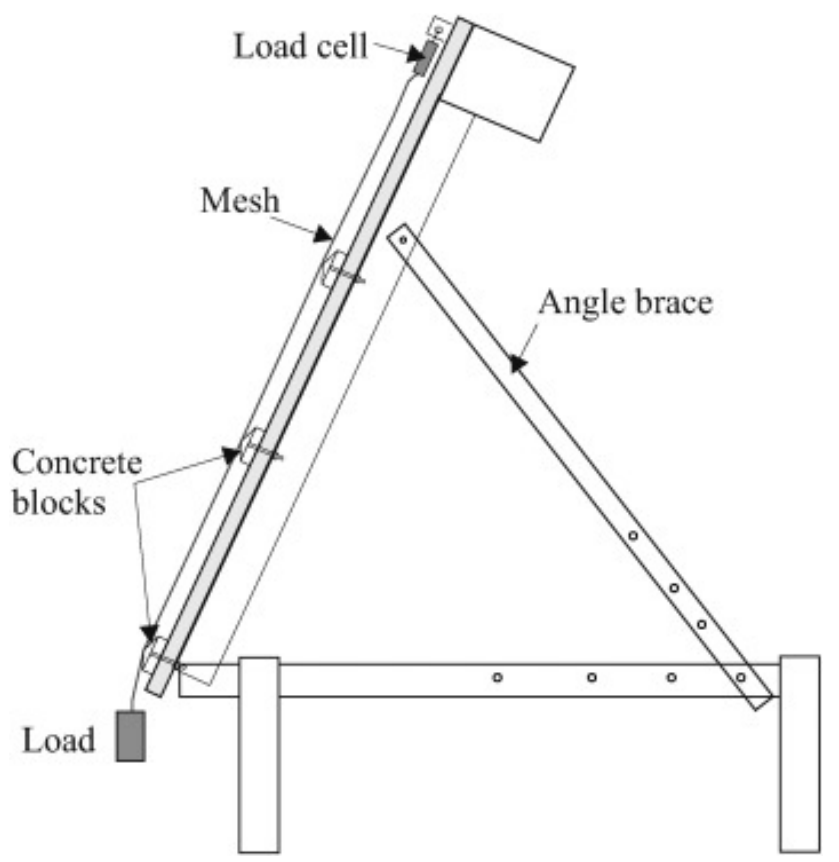


Figure 11. Experimental data plotted as the slope angle against the force reduction $\left(\mathrm{R}_{\mathrm{f}}\right)$ obtained for octahedron-shaped blocks: (a) 2 blocks, and (b) 8 blocks.
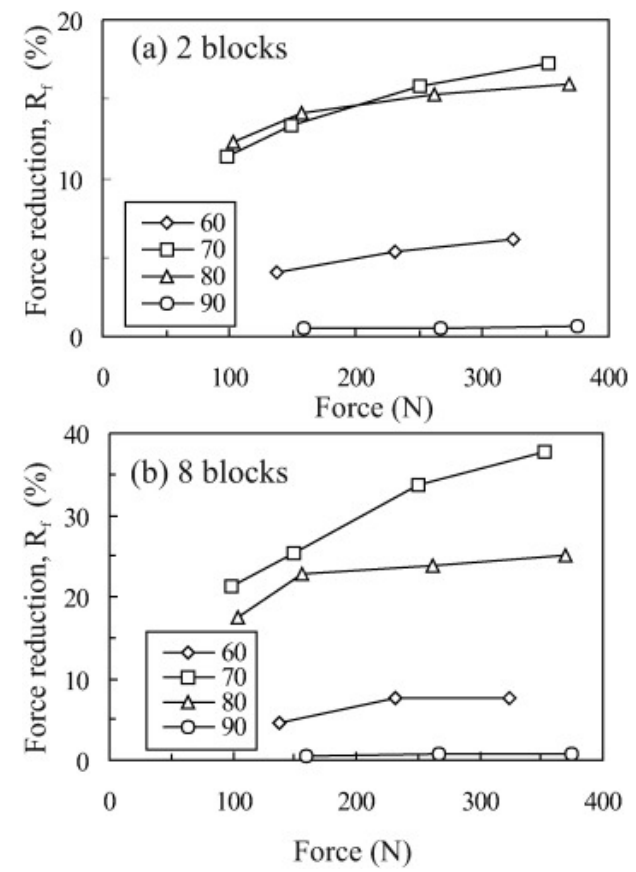

Figure 12. Test results obtained for the round-shaped blocks (a), and octahedron-shaped blocks (b) at the slope angle $(\beta)$ of $70^{\circ}$.
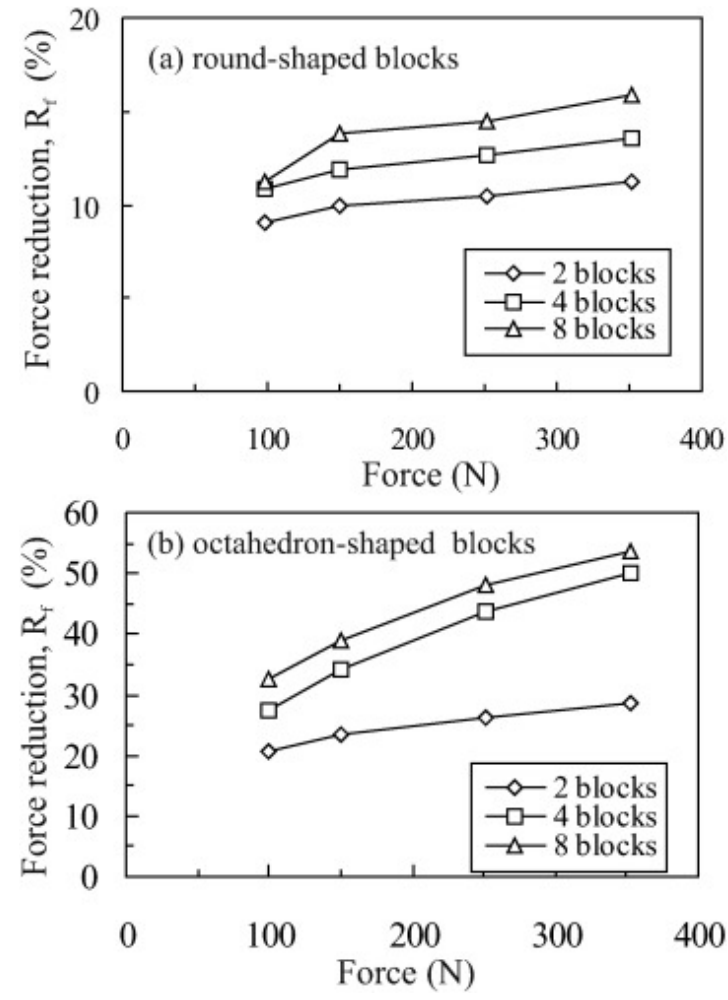
Figure 13. The relationship between the applied force and a) mobilized interface friction angle, and $b)$ the ratio between the interlocking $\left(F_{\text {int }}\right)$ and resistance $\left(F_{\text {res }}\right)$ forces.
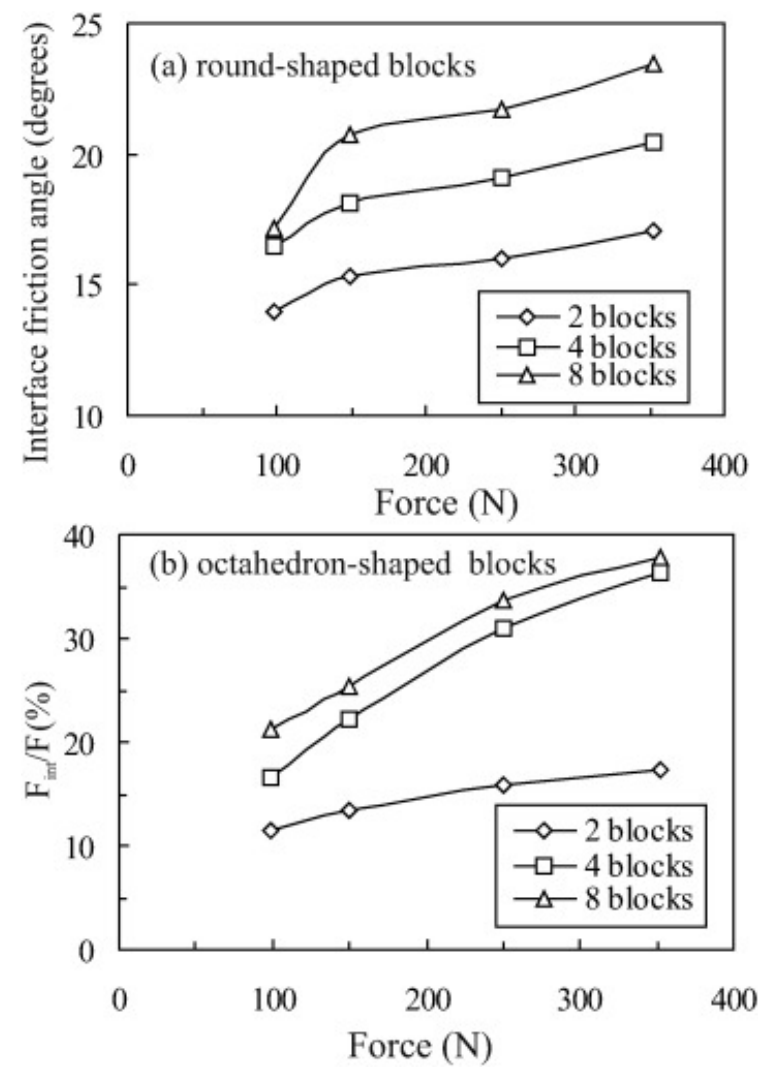